\title{
Comparison of Finite-Difference Computations of Natural Convection
}

\author{
K. E. Torrance* \\ Institute for Applied Technology, National Bureau of Standards, Washington, D.C. 20234
}

(August 16, 1968)

\begin{abstract}
Five numerical methods were compared for calculating two-dimensional, transient natural convection in an enclosure. Both implicit and explicit procedures were considered. Requirements for numerical stability were derived from analysis and experience, and when satisfied, the calculated flows for all methods were found to be similar. Consideration was also given to the accuracy and (energy and vorticity) conservation of the methods. One method was found to be conservative and stable without a restriction on the spatial mesh increment. This method can be successfully applied to nonlinear flows, but care must be exercised due to the presence of truncation errors which introduce false transport mechanisms.
\end{abstract}

Key Words: Enclosures; finite-difference; fluid flow; natural convection; numerical.

\section{Introduction}

\subsection{General}

The purpose of this paper is to compare the practical stability, truncation errors, and conservation properties of five finite-difference procedures for solving a problem in natural convection. Three of these methods are from the current literature $[2,6,20]^{1}$ and two are developed here. The ability of the various methods to produce physically meaningful solutions is examined; and solutions calculated with the various methods are compared.

The physical problem chosen for study is the natural convection flow induced in a vertical circular cylinder by a small hot spot centrally located on the floor (see fig. 1). Transient and steady laminar flows are considered in two-dimensional, axisymmetric cylindrical coordinates. Such a fluid motion is described by three simultaneous partial differential equations [4, 10]: (a) an equation relating stream function to vorticity, eq (2); (b) a time-dependent equation for vorticity, eq (3); and (c) a time-dependent equation for temperature, eq (4). The first equation is of elliptic type, and the last two are of parabolic type.

\subsection{Numerical Methods Tested}

This section provides a brief introduction to the five numerical methods tested. All methods employ successive over-relaxation [16, see chapter 11] for solving the finite-difference approximation of the elliptic equation, and no difficulties are encountered. The parabolic equations, on the other hand, contain nonlinear terms with first-order derivatives which express the influence of convection (hereafter called the convection terms). These terms introduce serious problems of stability and conservation into the finite-difference scheme. The numerical methods differ only in

${ }^{*}$ Research Associate from the Factory Mutual Engineering Corporation, Norwood, Massachusetts. Present address: Department of Thermal Engineering, Upson Hall, Cornell University, Ithaca, N.Y. 14850.

${ }^{1}$ Figures in brackets indicate the literature references at the end of this paper. 
their treatment of the parabolic equations, and these differences are highlighted in table 1. Methods IV and V are new and are modifications of methods II and I, respectively.

TABLE 1. Numerical methods applied to parabolic equations for temperature and vorticity Checks mean that the numerical methods possess the indicated properties.

\begin{tabular}{|c|c|c|c|c|c|c|c|c|}
\hline Method & Reference & $\begin{array}{c}\text { Explicit } \\
\text { or } \\
\text { implicit }\end{array}$ & Difference form for convection terms & $\begin{array}{c}\text { Order } \\
\text { of trun- } \\
\text { cation } \\
\text { error }\end{array}$ & $\begin{array}{l}\text { No time } \\
\text { step re- } \\
\text { striction }\end{array}$ & $\begin{array}{l}\text { No spatial } \\
\text { mesh size } \\
\text { restriction }\end{array}$ & $\begin{array}{l}\text { No false } \\
\text { diffusion } \\
\text { and con- } \\
\text { vection }\end{array}$ & $\begin{array}{c}\text { Satisfies } \\
\text { conser- } \\
\text { vation }\end{array}$ \\
\hline I..... & Barakat and Clark [2]....... & $\mathrm{E}$ & $2 \mathrm{pt}$ forward or backward... & $h$ & & 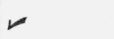 & & \\
\hline II... & Wilkes and Churchill [20]. & I & 3 pt central....................... & $h^{2}$ & & & $v$ & \\
\hline III. & Fromm [6]........................ & $\mathrm{E}$ & 3 pt central... & $h^{2}$ & & & $\checkmark$ & 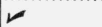 \\
\hline IV.. & Present study........ & I & 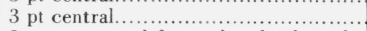 & $h^{2}$ & & & $\nu$ & 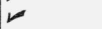 \\
\hline V... & Present study........ & $\mathrm{E}$ & 3 pt noncentral forward or backward.. & $\ddot{h}$ & & 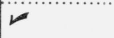 & & 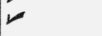 \\
\hline
\end{tabular}

An inspection of table 1 reveals that no one method is conservative, free of false transport, and stable without a restriction on the spatial mesh size. For conservation reasons, methods III-V are to be preferred over methods I and II. Of these, methods III and IV require a reduction in spatial mesh size with increasing flow velocities to achieve stability, while method $V$ does not. This mesh size restriction leads to prohibitively large demands on computer time and storage. The stability of method V, together with its conservation, are essential for calculating flows where the convection terms are important, as in the present problem. The introduction of false diffusion and convection, however, means that the calculated flows must be interpreted with care. In general, this false transport is important only in those flow regions where methods III and IV were found to break down.

Since the completion of this work, two additional differencing schemes have come to the author's attention [15, 1]. The first of these [15] was independently developed for steady flows and employs a differencing of the convection terms identical to method $\mathrm{V}$. The second method [1] has been employed for meteorological problems and was developed for the vorticity equation. This method conserves vorticity, mean kinetic energy, and mean square vorticity. The convection terms are differenced to $h^{2}$ accuracy, as with methods II-IV in table 1 , but conservation of mean square vorticity should lead to improved stability characteristics.

A comparison of the methods listed in table 1 is presented in the following sections. The physical problem is posed in section 2, some features and properties of the numerical methods are discussed in section 3, and comparative physical results are presented in section 4.

\section{Mathematical Description of the Physical Problem}

Consider the motion of a viscous fluid within a vertical circular cylinder of height $a$ and radius $b$ (see fig. 1). Erect a cylindrical coordinate system $(x, r)$ with origin at the center of the base. The flow is assumed to be axisymmetric with no variations in the azimuthal direction. The fluid is initially motionless and at a uniform temperature $T_{0}$. The enclosure walls are also at this temperature, except for a small centrally located circular spot on the base of radius $c$ which is at a temperature $T_{h}>T_{0}$. The temperature difference $T_{h}-T_{0}$ initiates and sustains the natural convection flow within the enclosure.

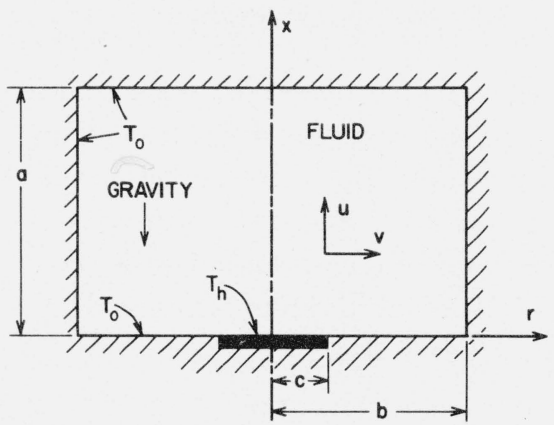

FigURE 1. Cylindrical enclosure and coordinate system. 
The Boussinesq approximation is used [4, see p. 16]; in this density $(\rho)$ is assumed constant except for the generation of buoyancy forces. Other fluid properties are taken as constant: kinematic viscosity $(\nu)$, thermal diffusivity $(\kappa)$, and volume expansion coefficient $(\beta)$. We introduce the following dimensionless quantities: time, $\tau=\left(\kappa / a^{2}\right) t$; vertical and radial coordinates, $X=x / a$ and $R=r / a$; vertical and radial components of velocity, $U=(a / \kappa) u$ and $V=(a / \kappa) v$; and temperature $\Theta=\left(T-T_{0}\right) /\left(T_{h}-T_{0}\right)$.

The governing equations in dimensionless form are

$$
\begin{gathered}
U=\frac{1}{R} \frac{\partial \Psi}{\partial R}, V=-\frac{1}{R} \frac{\partial \Psi}{\partial X}, \\
-\Omega=\frac{1}{R} \frac{\partial^{2} \Psi}{\partial X^{2}}+\frac{\partial}{\partial R}\left(\frac{1}{R} \frac{\partial \Psi}{\partial R}\right), \\
\frac{\partial \Omega}{\partial \tau}+\left[\frac{\partial(U \Omega)}{\partial X}+\frac{\partial(V \Omega)}{\partial R}\right]=-G r r^{2} \frac{\partial \Theta}{\partial R}+\operatorname{Pr}\left[\frac{\partial^{2} \Omega}{\partial X^{2}}+\frac{\partial}{\partial R}\left(\frac{1}{R} \frac{\partial(R \Omega)}{\partial R}\right)\right],
\end{gathered}
$$

and

$$
\frac{\partial \Theta}{\partial \tau}+\left[\frac{\partial(U \Theta)}{\partial X}+\frac{1}{R} \frac{\partial(R V \Theta)}{\partial R}\right]=\left[\frac{\partial^{2} \Theta}{\partial X^{2}}+\frac{1}{R} \frac{\partial}{\partial R}\left(R \frac{\partial \Theta}{\partial R}\right)\right] .
$$

Equation (3) contains the Prandtl number $\operatorname{Pr}=\nu / \kappa$ and the Grashof number $G r=g \beta\left(T_{h}-T_{0}\right) a^{3} / \nu^{2}$. The latter number is used for natural convection flows and denotes the product of buoyancy and inertia forces divided by the square of the viscous force. The acceleration of gravity is denoted by $g$. The existence of a stream function $\Psi$ is assumed such that the velocities are given by (1). The mass conservation equation $\operatorname{div} \bar{U}=0$ is then automatically satisfied. The vorticity vector $\bar{\Omega}=\operatorname{curl} \bar{U}$ has only an azimuthal component, $\Omega=\partial V / \partial X-\partial U / \partial R$, given by (2). The conservation equations for vorticity and energy are (3) and (4) respectively. In these equations, bracketed terms on the left and right sides respectively denote the convective and diffusive transport terms. The term containing $\mathrm{Gr}$ in (3) represents the vorticity source due to buoyancy.

The convective terms in eqs (3) and (4) are in a "conservation" form [3,6] suitable for methods III, IV, and V of table 1. Altemate "nonconservation" forms for these terms are utilized for methods I and II. The alternate equations are obtained by introducing a modified vorticity $\Omega^{\prime}=(1 / R) \Omega$ into (2) and (3), followed by subtraction of $\Omega^{\prime} \operatorname{div} \bar{U}=0$ and $\Theta \operatorname{div} \bar{U}=0$ from (3) and (4) respectively. In terms of modified vorticity $\Omega^{\prime}$, eqs (1) through (4) become

$$
\begin{gathered}
U=\frac{1}{R} \frac{\partial \Psi}{\partial R}, V=-\frac{1}{R} \frac{\partial \Psi}{\partial X}, \\
-R \Omega^{\prime}=\frac{1}{R} \frac{\partial^{2} \Psi}{\partial X^{2}}+\frac{\partial}{\partial R}\left(\frac{1}{R} \frac{\partial \Psi}{\partial R}\right), \\
\frac{\partial \Omega^{\prime}}{\partial \tau}+U \frac{\partial \Omega^{\prime}}{\partial X}+V \frac{\partial \Omega^{\prime}}{\partial R}=-\frac{G r P r^{2}}{R} \frac{\partial \Theta}{\partial R}+\operatorname{Pr}\left[\frac{\partial^{2} \Omega^{\prime}}{\partial X^{2}}+\frac{1}{R} \frac{\partial}{\partial R}\left(\frac{1}{R} \frac{\partial\left(R^{2} \Omega^{\prime}\right)}{\partial R}\right)\right],
\end{gathered}
$$

and

$$
\frac{\partial \Theta}{\partial \tau}+U \frac{\partial \Theta}{\partial X}+V \frac{\partial \Theta}{\partial R}=\frac{\partial^{2} \Theta}{\partial X^{2}}+\frac{1}{R} \frac{\partial}{\partial R}\left(R \frac{\partial \Theta}{\partial R}\right) .
$$

Equations (1)-(4) and $\left(1^{\prime}\right)-\left(4^{\prime}\right)$ are subject to initial conditions and boundary conditions. The initial conditions are:

$$
\Omega=\Theta=0 \text { for } \quad \tau<0,0 \leqslant X \leqslant 1 \text {, and } 0 \leqslant R \leqslant R_{b} \text {. }
$$


The boundary conditions ${ }^{2}$ for $\tau \geqslant 0$ are:

$$
\begin{array}{lll}
\Psi=\partial \Psi / \partial X=0 & \text { for } X=0, & 0 \leqslant R \leqslant R_{b} ; \\
\Theta=1 & \text { for } X=0, & 0 \leqslant R<R_{c} ; \\
\Theta=0.5 & \text { for } X=0, & R=R_{c} ; \\
\Theta=0 & \text { for } X=0, & R_{c}<R \leqslant R_{b} \\
\Psi=\partial \Psi / \partial X=\Theta=0 & \text { for } X=1, & \text { all } R ; \\
\Psi=\Omega=\partial \Theta / \partial R=0 & \text { for } R=0, & \text { all } X ; \text { and } \\
\Psi=\partial \Psi / \partial R=\Theta=0 & \text { for } R=R_{b}, & \text { all } X .
\end{array}
$$

For equations $\left(1^{\prime}\right)-\left(4^{\prime}\right)$ replace $\Omega=0$ in (5) by $\Omega^{\prime}=0$, and $\Omega=0(R=0$, all $X)$ in (6) by the dual conditions $V=\partial U / \partial R=0 .^{3}$

The boundary conditions introduce two geometric parameters: the aspect ratio of the enclosure (radius/height) $R_{b}=b / a$ and the relative size of the heat source (heat source radius/enclosure height) $R_{c}=c / a$.

To determine $\theta$ and $\Omega^{\prime}$ along the centerline, special forms of the conservation equations are needed to avoid an indeterminate form as $R \rightarrow 0$. By incorporating boundary conditions at $R=0$ and using L'Hospital's rule, eqs $(4),\left(3^{\prime}\right)$, and $\left(4^{\prime}\right)$ respectively reduce to

$$
\begin{aligned}
& \frac{\partial \Theta}{\partial \tau}+\frac{\partial(\boldsymbol{U})}{\partial X}+\frac{\partial^{2}(R V \Theta)}{\partial R^{2}}=\frac{\partial^{2} \Theta}{\partial X^{2}}+2 \frac{\partial^{2} \Theta}{\partial R^{2}}, \\
& \frac{\partial \Omega^{\prime}}{\partial \tau}+U \frac{\partial \Omega^{\prime}}{\partial X}=-G r P r^{2} \frac{\partial^{2} \Theta}{\partial R^{2}}+\operatorname{Pr}\left[\frac{\partial^{2} \Omega^{\prime}}{\partial X^{2}}+4 \frac{\partial^{2} \Omega^{\prime}}{\partial R^{2}}\right],
\end{aligned}
$$

and

$$
\frac{\partial \Theta}{\partial \tau}+U \frac{\partial \Theta}{\partial X}=\frac{\partial^{2} \Theta}{\partial X^{2}}+2 \frac{\partial^{2} \Theta}{\partial R^{2}}
$$

For convenience, the velocities $U$ and $V$ are retained explicitly in the problem formulation. However, an examination of eqs (1)-(7) (or alternately, eqs $\left(1^{\prime}\right)-\left(7^{\prime}\right)$ ) reveals that the essential dependent variables are $\Psi, \Omega$, and $\Theta$ (or $\Psi, \Omega^{\prime}$, and $\theta$ ), while the independent variables are $X, R$, and $\tau$. The parameters of the problem are $R_{b}, R_{c}, \operatorname{Pr}$, and $G r$. Throughout this study, the aspect ratio, relative heat source size, and Prandtl number are held fixed at $R_{b}=1, R_{c}=0.1$ and $\operatorname{Pr}=0.7$, respectively. The Grashof number $G r$ is assumed equal to $1 \times 10^{5}$, except in section 4.4, which considers larger values of this parameter.

\section{Numerical Methods, Formulation and Some Properties}

\subsection{Grid System and Calculation Sequence}

An approximate solution of eqs $(1)-(7)$ or $\left(1^{\prime}\right)-\left(7^{\prime}\right)$ will be obtained at a finite number of grid points having coordinates $X=i \Delta X, R=j \Delta R$, and at discrete times $\tau_{n}$, where $i, j$, and $n$ are integers. The grid spacings in the $X$ and $R$ directions are denoted by $\Delta X$ and $\Delta R$. The symbol $\tau_{n}$ denotes the time level after the $n$th time step $\Delta \tau_{n}$. The values of $\Psi, \Omega, \Theta, U$, and $V$ at each grid point should be thought of as average values over a small volume of fluid surrounding the point.

\footnotetext{
${ }^{2}$ Note that explicit boundary conditions for vorticity on the solid boundaries are not available. This causes no difficulty with the explicit solution methods and only slight difficulty with the implicit methods.

${ }^{3}$ The centerline boundary condition $\Omega=R \Omega^{\prime}=0$ is satisfied for all finite values of $\Omega^{\prime}$. Although the vorticity $\Omega$ is zero, the modified vorticity $\Omega^{\prime}$ is not, in general, zero. Barakat and Clark [2] used the modified vorticity $\Omega^{\prime}$ and assumed $\Omega^{\prime}=0$ along the centerline. This is incorrect, and may have contributed to the oscillations in stream function (near the centerline) and heat transfer observed in their calculations. The vorticity $\Omega^{\prime}$ on the centerline can be evaluated with the aid of eq ( $7^{\prime}$ a).
} 
All of the numerical methods listed in table 1 advance the fields of $\Psi, \Omega$, and $\Theta$ across a time step in the same sequence. All quantities are assumed known at a time $\tau_{n}$. The sequence for advancement from time $\tau_{n}$ to the new level $\tau_{n+1}=\tau_{n}+\Delta \tau$ is as follows:

1. Temperature $(\Theta)$ at all interior grid points is advanced with suitable difference approximations of (4) and (7) or (4') and (7'b). This is discussed in section 3.2.

2. Vorticity $\left(\Omega\right.$ or $\left.\Omega^{\prime}\right)$ at all interior grid points is similarly advanced with approximations of (3) or $\left(3^{\prime}\right)$ and $\left(7^{\prime}\right.$ a). This is discussed in section 3.2 .

3. Stream function $(\Psi)$ at all interior grid points is brought up to date with the new vorticity field by using a difference approximation of $(2)$ or $\left(2^{\prime}\right)$. This is discussed in section 3.3 .

4. The vorticity on the solid boundaries is determined from the new stream function field, as are the velocities $U$ and $V$. This is discussed in section 3.3.

5. The field of mesh points is scanned in order to determine the time step $\Delta \tau$ for the next time advancement. This is limited by practical stability requirements and is discussed in section 3.4.

\subsection{Parabolic Equations}

\section{a. Differencing Schemes}

The approximations of the equations for temperature and vorticity (for steps 1 and 2 above) are described in conventional notation in table 2 . In this section we comment on the distinguishing features and some of the properties of these schemes.

TABLE 2. Finite-difference approximations of the parabolic equations for temperature and vorticity Illustrated with the simplified temperature equations, $\frac{\partial \Theta}{\partial \tau}+U \frac{\partial \Theta}{\partial X}-\frac{\partial^{2} \Theta}{\partial X^{2}}=0$ (for methods I and II) and $\frac{\partial \Theta}{\partial \tau}+\frac{\partial(U \Theta)}{\partial X}-\frac{\partial^{2} \Theta}{\partial X^{2}}=0$ (for methods III-V).

\begin{tabular}{|c|c|c|c|c|}
\hline \multicolumn{2}{|c|}{ Method } & $\frac{\partial \Theta}{\partial \tau}$ & $U \frac{\partial \Theta}{\partial X}$ or $\frac{\partial(U \Theta)}{\partial X}$ & $\frac{\partial^{2} \theta}{\partial X^{2}}$ \\
\hline \multicolumn{2}{|l|}{ I } & $\frac{\Theta_{i, j}^{n+1}-\theta_{i, j}^{n}}{\Delta \tau}+0(\Delta \tau)$ & 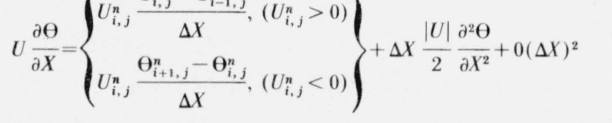 & $\frac{\Theta_{i+1, j}^{n}-2 \Theta_{i, j}^{n}+\Theta_{i-1, j}^{n}}{(\Delta X)^{2}}+0(\Delta X)^{2}$ \\
\hline \multirow{2}{*}{ II } & a & $\frac{\Theta_{i, j}^{n+1 / 2}-\Theta_{i, j}^{n}}{\Delta \tau / 2}+0(\Delta \tau)$ & $U \frac{\partial \Theta}{\partial X}=U_{i, j}^{n} \frac{\Theta_{i+1, j}^{n}-\Theta_{i-1, j}^{n}}{2 \Delta X}+0(\Delta X)^{2}$ & $\frac{\Theta_{i+1, j}^{n}-2 \Theta_{i, j}^{n}+\Theta_{i-1, j}^{n}}{(\Delta X)^{2}}+0(\Delta X)^{2}$. \\
\hline & b & $\frac{\Theta_{i, j}^{n+1}-\Theta_{i, j}^{n+1 / 2}}{\Delta \tau / 2}+0(\Delta \tau)$ & $U \frac{\partial \Theta}{\partial X}=U_{i, j}^{n} \frac{\Theta_{i+1, j}^{n+1}-\Theta_{i-1, j}^{n+1}}{2 \Delta X}+0(\Delta X)^{2}+0\left(\frac{\Delta \tau}{\Delta X}\right)$ & $\frac{\Theta_{i+1, j}^{n+1}-2 \Theta_{i, j}^{n+1}+\Theta_{i-1, j}^{n+1}}{(\Delta X)^{2}}+0(\Delta X)^{2}+0\left(\frac{\Delta \tau}{\Delta X}\right)$. \\
\hline \multicolumn{2}{|c|}{ III } & $\frac{\Theta_{i, j}^{n+1}-\Theta_{i, j}^{n-1}}{2 \Delta \tau}+0(\Delta \tau)^{2}$ & $\frac{\partial(U \boldsymbol{\theta})}{\partial X}=\frac{U_{i+1, j}^{n} \Theta_{i+1, j}^{n}-U_{i-1, j}^{n} \theta_{i-1, j}^{n}}{2 \Delta X}+0(\Delta X)^{2}$ & $\begin{aligned} \frac{\Theta_{i+1, j}^{n}-\Theta_{i, j}^{n+1}-\Theta_{i, j}^{n-1}+\Theta_{i-1, j}^{n}}{(\Delta X)^{2}} & \\
& +0(\Delta X)^{2}+0\left(\frac{\Delta \tau}{\Delta X}\right)^{2} .\end{aligned}$ \\
\hline \multirow{2}{*}{ IV } & $\mathrm{a}$ & Same as method Ila. & $\frac{\partial(U \theta)}{\partial X}=\frac{U_{i+1, j}^{n} \Theta_{i+1, j}^{n}-U_{i-1, j}^{n} \Theta_{i-1, j}^{n}}{2 \Delta X}+0(\Delta X)^{2}$ & Same as method IIa. \\
\hline & b & Same as method IIb. & $\frac{\partial(U \Theta)}{\partial X}=\frac{U_{i+1, j}^{n} \Theta_{i+1, j}^{n+1}-U_{i-1, j}^{n} \Theta_{i-1, j}^{n+1}}{2 \Delta X}+0(\Delta X)^{2}+0\left(\frac{\Delta \tau}{\Delta X}\right)$ & Same as method IIb. \\
\hline$V$ & & Same as method I. & 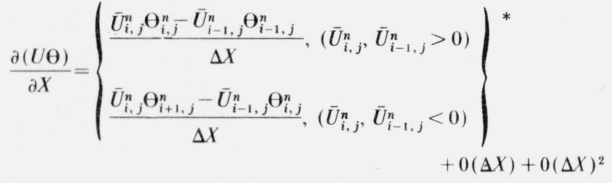 & Same as method I. \\
\hline
\end{tabular}

${ }^{*} \bar{U}_{m, n}=\left(U_{m+1, n}+U_{m, n}\right) / 2$.

Application of the various numerical methods to the parabolic equations is illustrated with the one-space dimension temperature equation 


$$
\frac{\partial \Theta}{\partial \tau}+\frac{\partial(U \boldsymbol{\theta})}{\partial X}-\frac{\partial^{2} \Theta}{\partial X^{2}}=0
$$

in place of eqs (3), (4), and (7); and

$$
\frac{\partial \Theta}{\partial \tau}+U \frac{\partial \Theta}{\partial X}-\frac{\partial^{2} \Theta}{\partial X^{2}}=0
$$

in place of eqs $\left(3^{\prime}\right),\left(4^{\prime}\right)$, and $\left(7^{\prime}\right)$. Equation (8) is employed for methods III-V and eq $\left(8^{\prime}\right)$ for methods I and II (see table 2). It is understood that the discussion and differencing of each term of these equations applies to similar terms in the two-space dimension temperature and vorticity equations.

Methods I, III, and V are explicit procedures for calculating quantities at time $n+1$ in terms of their values at times $n$ and $n-1$. Methods II and IV are two step implicit procedures (see [12, $16,20]$ ), going from time $n$ to $n+1 / 2$ in step a, and from $n+1 / 2$ to $n+1$ in step $b$. During the first half time step (or second half time step), the $X$ and $R$ space derivatives are respectively approximated at time levels $n$ and $n+1 / 2$ (or $n+1$ and $n+1 / 2$ ). Clearly, the time steps $a$ and $b$ are implicit in the $R$ and $X$ directions, respectively. This is called an alternating direction, implicit scheme and leads to a tridiagonal matrix of unknown temperatures or vorticities for each $R$-row or $X$-column, as the case may be. These matrices are readily inverted by a simple algorithm.

Column two of table 2 lists the approximation of the derivative $\partial \Theta / \partial \tau$. A central time difference is used with method III, and forward time differences with all others. The last column of the table lists the approximation of the diffusion terms, $\partial^{2} \Theta / \partial X^{2}$. Three point space differences are used for all methods.

The approximations of the convection terms $U \partial \Theta / \partial X$ or $\partial(U \Theta) / \partial X$ are listed in column three. At any grid point the velocities are evaluated at time $n$ and are treated as constants over the time step. With method I, the convection terms are approximated with two-point forward or backward differences as the coefficient velocity $U$ is positive or negative, respectively. Methods II-IV all employ three-point central differences for these terms. Method V employs a modified form of forward or backward differences in which the mean velocity $\bar{U}_{n, m}$ in the numerator is multiplied by $\Theta_{n, m}$ or $\Theta_{n+1, m}$ as $\bar{U}_{n, m}$ is positive or negative, respectively. If the two mean velocity coefficients are of different sign, one term from the numerator of each of the two approximations shown is required.

Two points about the vorticity equation should be noted. First, this equation contains a buoyancy source term which is not listed in table 2. The term contains $\partial \Theta / \partial R$, which is approximated with three-point central differences. Method III evaluates the term at time $n$, all other methods evaluate it at time $n+1$. Second, when the implicit methods II and IV are applied to the vorticity equation, it is necessary to temporarily assume the vorticity distribution on the solid walls at times $n+1$ and $n+1 / 2$ equal to that at time $n$. Thus, the wall vorticity is out of step with the advancement of the interior vorticity field. The error introduced by this assumption decreases with both small time steps and the approach to steady state.

The rest of this subsection presents brief comments on the stability and conservation properties of the parabolic difference equations. Additional discussion is provided in sections 3.4 and 4.3 , respectively.

The stability of the various numerical methods is principally due to some key approximations shown in table 2. For methods $\mathrm{I}$ and $\mathrm{V}$, it is the use of forward or backward differencing for the convection terms [2, 7, 14 (see p. 194)]. For method III, it is the duFort-Frankel differencing [5] of the diffusion terms $\partial^{2} \Theta / \partial X^{2}$. For methods II and IV, it is the alternating direction, implicit nature of the scheme (due to Peaceman-Rachford [12, 16 (see p. 366), 20]).

A lengthy but straightforward study of methods $\mathrm{I}-\mathrm{V}$ shows that conservation is satisfied for III-V, but not for I and II. The elements of such a study are presented in [3,6]. Conservation of energy or vorticity within the grid system exists if the difference equations for temperature or vorticity are summed over all interior grid points and no spurious sources or sinks of these quan- 
tities are found. The convection and diffusion terms cancel in pairs. The net energy or vorticity transport from the wall mesh points into the enclosure just balances the net increase of energy or vorticity within the mesh system. Heat transfer rates to and from the enclosure can thus be determined by calculating the transfer from wall mesh points. If conservation is satisfied, there is no net increase of energy within the enclosure at steady state, and the total heat added to the enclosure balances the heat removed.

\section{b. Truncation Errors}

The truncation errors shown in table 2 are worthy of some discussion. These estimates are obtained by Taylor series analysis as described in references [5, 11, 13]. Methods II-IV are most easily discussed and are treated in the first paragraph. Methods I and V are more complicated, and are discussed in the second and third paragraphs, respectively.

For methods II-IV, all space derivatives are approximated to $(\Delta X)^{2}$ accuracy. The use of multiple time levels in conjunction with these space derivatives, however, introduces additional truncation errors of $0(\Delta \tau / \Delta X)$ for methods II and IV and of $0(\Delta \tau / \Delta X)^{2}$ for method III. Substitution of the difference approximations listed in table 2 into eqs (8) or $\left(8^{\prime}\right)$, as appropriate, introduces truncation errors of $0(\Delta \tau)+0(\Delta \tau / \Delta X)+0(\Delta X)^{2}$ for methods II and IV and of $0(\Delta \tau)^{2}+0(\Delta \tau / \Delta X)^{2}$ $+0(\Delta X)^{2}$ for method III. The coefficients of these terms involve derivatives of higher order than those of the basic eqs (8) or $\left(8^{\prime}\right)$. Stability requires that $\Delta \tau \ll \Delta X$, and when $\Delta X$ is small, these truncation errors are usually neglected.

With method $\mathrm{I}$, the $0(\Delta X)$ approximation of the convection term $U \partial \Theta / \partial X$ introduces a large truncation error. A Taylor series expansion for $\Theta_{i-1, j}$ about $\Theta_{i, j}$ can be rearranged to solve for $(\partial \Theta / \partial X)_{i, j}$

$$
\left(\frac{\partial \Theta}{\partial X}\right)_{i, j}=\frac{\Theta_{i, j}-\Theta_{i-1, j}}{\Delta X}+\left\{\frac{\Delta X}{2}\left(\frac{\partial^{2} \Theta}{\partial X^{2}}\right)_{i, j}-\frac{(\Delta X)^{2}}{6}\left(\frac{\partial^{3} \Theta}{\partial X^{3}}\right)_{i, j}+\ldots\right\}
$$

The first term on the right side is a backward difference. The term in braces is the truncation error. Multiplication of eq (9) by $U_{i, j}\left(U_{i, j}>0\right)$ leads to the form shown in the table. The solution of the difference equation (i.e., the approximation of $\left.\left(8^{\prime}\right)\right)$ is equivalent to a solution of the differential equation

$$
\frac{\partial \Theta}{\partial \tau}+U \frac{\partial \Theta}{\partial X}-\left(1+\Delta X \frac{|U|}{2}\right) \frac{\partial^{2} \Theta}{\partial X^{2}}=0(\Delta \tau)+0(\Delta X)^{2} .
$$

The coefficients of the terms $0(\Delta \tau)$ and $0(\Delta X)^{2}$ involve derivatives of higher order than appear in $\left(8^{\prime}\right)$, and for reasons noted in the preceding paragraph, these truncation errors are usually neglected. The differencing of the convection terms in equation $\left(8^{\prime}\right)$ introduces an additional, or false, heat diffusivity $\Delta X \frac{|U|}{2}$. Only for small $\Delta X \frac{|U|}{2}$ does the difference approximation approach the differential equation $\left(8^{\prime}\right)$. For large $\Delta X \frac{|U|}{2}$, care must be exercised because a false heat diffusivity is introduced (or a false viscosity in the case of the vorticity equation). ${ }^{4}$

Turning now to method $\mathrm{V}$, the analysis is similar to that presented for method I above. The truncation error of $0(\Delta X)$ in the convection term $\partial(U \Theta) / \partial X$ is more complicated, however. It can be shown that the solution of the difference equation (i.e., the approximation of (8)) is equivalent to a solution of the differential equation

$$
\frac{\partial \Theta}{\partial \tau}+\left\{\frac{\partial(U \Theta)}{\partial X}-\frac{\Delta X}{2} \frac{\partial U}{\partial X} \frac{\partial \Theta}{\partial X}\right\}-\left\{1+\Delta X \frac{\bar{U}_{i, j}}{2}\right\} \frac{\partial^{2} \Theta}{\partial X^{2}}=0(\Delta \tau)+0(\Delta X)^{2}
$$

'This problem cannot be overcome by eliminating $\partial^{2} \Theta / \partial X^{2}$ from (9) with, for example, three-point central differences. If this is done, eq (9) becomes a three-point central difference approximation of $\partial \Theta / \partial X$, with its associated stability problems. 
when $\bar{U}_{i, j}, \bar{U}_{i-1, j}>0$. Again, the terms on the right side are usually neglected. There is now a false heat diffusion (or a false viscosity) as well as a false convection. The latter is a subtraction from the real convection and is small whenever $\frac{\Delta X}{2}(\partial U / \partial X)(\partial \theta / \partial X)$ is small. When this last term and $\Delta X \frac{U}{2}$ are small, the difference approximation approaches the differential equation (8). When these terms are large, care must be exercised.

\subsection{Elliptic Equation, Velocities, and Wall Vorticity}

The computations described in steps 3 and 4 of section 3.1 are identical for all five numerical methods, and are integrated and presented in this section. Equations for the vorticity $\Omega$ (used for methods III-V) are considered here. Appropriate equations in terms of modified vorticity $\Omega^{\prime}$ (for methods I and II) are obtained by the substitution $\Omega=R \Omega^{\prime}$. Step 3 is considered first.

The elliptic equation (2) relates stream function to vorticity at any given time. The new vorticities $\Omega_{i, j}^{n+1}$ are introduced and the space derivatives are approximated by three-point central differerences with truncation errors of $0(\Delta X)^{2}$ and $0(\Delta R)^{2}$. An iterative technique known as successive over-relaxation [21] was employed to obtain the new stream function field. No problems of stability or conservation are encountered. Thus, if $\Psi_{i, j}^{(s)}$ denotes the approximate stream function at a point interior to the solid boundaries and centerline after $s$ iterations, a further approximation $\Psi_{i, j}^{(s+1)}$ is obtained from

$$
\begin{aligned}
& \Psi_{i, j}^{(s+1)=(1-\omega) \Psi_{i, j}^{(s)}+}+\frac{\omega}{\frac{2}{(\Delta X)^{2}}+\frac{1}{(\Delta R)^{2}}\left(\frac{j}{j+\frac{1}{2}}+\frac{j}{j-\frac{1}{2}}\right)}\left\{j \Delta R \Omega_{i, j}^{n+1}+\frac{1}{(\Delta X)^{2}}\left[\Psi_{i+1, j}^{(s)}+\Psi_{i-1, j}^{(s+1)}\right]\right. \\
&\left.+\frac{1}{(\Delta R)^{2}}\left[\frac{j}{j+\frac{1}{2}} \Psi_{i, j+1}^{(s)}+\frac{j}{j-\frac{1}{2}} \Psi_{i, j-1}^{(s+1)}\right]\right\}
\end{aligned}
$$

It is understood that all values of $\Psi$ in this equation pertain to time $n+1$. The optimum value of the relaxation parameter $\omega$ was calculated for a given system of grid points (see eqs (1.9) and (4.6) of reference [21]). ${ }^{5}$

Successive sweeps of eq (12) over the field of mesh points were alternated in the vertical and radial directions. Iteration was terminated when

$$
\frac{\max _{i, j}\left|\Psi_{i, j}^{(s+1)}-\Psi_{i, j}^{(s)}\right|}{\max _{i, j}\left|\Psi_{i, j}^{(s+1)}\right|}<0.0001 .
$$

The number 0.0001 was arrived at by experimentation, noting that more stringent criteria led to no essential difference in the solution of test problems. The numerator on the left side of (13) is the maximum change in stream function occurring in the field of grid points as a result of one approximation step. The denominator is the maximum value of stream function in the field.

In practice, the most time consuming part of the whole calculation was the iteration of eq (12) at each time step. One iterative sweep took almost as long as the entire computation of the new temperature or vorticity field described in the previous section. The following procedures resulted in a considerable savings in computer time. A maximum number of iterations, $s_{\max }$, was permitted. For 100,400 , and 1000 mesh points, $s_{\max }$ was 20,25 , and 35 , respectively. The convergence criterion (13) was generally achieved within $s_{\max }$ iterations after the initial flow transient was over. The stream function field was then updated less frequently, typically every $1+0.25\left(s_{\text {max }}-s_{\text {conversence }}\right)$ time steps.

${ }^{5}$ An independent determination of the optimum $\omega$ was made with the aid of a computer. Equation (12) was solved for various $\omega$ starting with a given $\Omega$ field. The value of $\omega$ leading to fastest convergence was sufficiently close to the calculated value so that the latter was used. 
The computations described in step 4 (sec. 3.1) are discussed in the rest of this section. The numerical approximations up to this point, together with the boundary conditions (6), provide the entire fields of $\Psi$ and $\theta$, and the field of $\Omega$ interior to the solid boundaries, all at time $n+1$. Still to be calculated at time $n+1$ are the unknown values of vorticity $\Omega$ on the solid boundaries ${ }^{6}$ and the velocity fields $U$ and $V$. The new stream function field is used for this purpose.

The vorticity on the solid boundaries is obtained by first reducing eq (2) to $\Omega=-(1 / R) \partial^{2} \Psi / \partial X^{2}$ for $X=0$ or $X=1$, and to $\Omega=-\left(1 / R_{b}\right) \partial^{2} \Psi / \partial R^{2}$ for $R=R_{b}$. The second order derivatives are approximated by expanding $\Psi$ in a Taylor series about the wall. Expansions for the two grid points nearest the wall, together with the boundary conditions on $\Psi$, yield wall vorticity approximations such as the following, which applies when $X=0$ :

$$
\Omega_{0, j}^{n+1}=-\frac{8 \Psi_{1, j}-\Psi_{2, j}}{2 j \Delta R(\Delta X)^{2}}+0(\Delta X)^{2} .
$$

Finally, the fields of $U$ and $V$ are calculated with three point central difference approximations of eqs (1). These approximations (of $0(\Delta X)^{2}$ and $0(\Delta R)^{2}$ ) are not presented here, but it is readily verified that they automatically satisfy a central difference approximation of the continuity equation, $\operatorname{div} \bar{U}=0$. The conservation of mass within the grid system is thereby established.

\subsection{Practical Stability Considerations for the Parabolic Equations}

At this point, the fields of $\Psi, \Omega, \Theta, U$, and $V$ are current at time $n+1$ for all methods. Further integration of the parabolic temperature and vorticity equations in time and space requires consideration of practical stability. This is step 5 of section 3.1. Practical stability imposes a restriction on the size of the time step $\Delta \tau$ for methods I and $V$ (eqs (19) and (20), respectively) and restrictions on $\Delta \tau, \Delta X$, and $\Delta R$ for methods II-IV (eqs (15)). The size of $\Delta \tau$ is calculated from the appropriate equations (assuming for methods II-IV that the restrictions on $\Delta X$ and $\Delta R$ are satisfied). The fields of $\Psi, \Omega, \Theta, U$, and $V$ are then advanced across this time step by repeating the whole cycle described in sections 3.2 and 3.3 .

A complete analysis of the nonlinear equations to determine the exact form of the stability requirements is not always possible. Such is the case for methods II-IV. The linearized stability analysis of von Neumann [11] has been applied to methods II [19] and III [6] and leads only to a restriction on $\Delta \tau$ for method III. Numerical experience with methods II-IV for a limited range of flows suggests that the following empirical restrictions be applied at each grid point for integration of the temperature and vorticity equations:

$$
\begin{gathered}
\Delta X \lesssim \frac{8}{\left|U_{i, j}\right|}, \Delta R \lesssim \frac{8}{\left|V_{i, j}\right|}, \\
\Delta \tau \leqslant\left[\frac{2}{(\Delta X)^{2}}+\frac{2}{(\Delta R)^{2}}\right]^{-1},
\end{gathered}
$$

and

$$
\Delta \tau \leqslant\left[\frac{\left|U_{i, j}\right|}{\Delta X}+\frac{\left|V_{i, j}\right|}{\Delta R}\right]^{-1}
$$

Note the restriction on spatial mesh size (15a), which presumably results from the use of three point central differences for the three methods. Within limited ranges these restrictions have led to stability, but they cannot be regarded as general. In particular, during flow transients with large initial changes, time steps smaller than suggested by $(15 \mathrm{~b})$ or $(15 \mathrm{c})$ were required.

${ }^{6}$ This delay in calculating the wall vorticities causes no difficulty with the explicit methods I, III, and V. For the implicit methods II and IV, it was necessary in section $3.2 \mathrm{a}$ to temporarily assume that the wall vorticities at times $n+1$ and $n+1 / 2$ were equal to their values at time $n$. 
Analytical stability criteria are possible for methods I and V. The analysis is applicable to explicit, nonlinear, two time level differencing schemes and is discussed in reference [2]. This paragraph is based on that analysis. To be specific, stability of the integration of the temperature eqs (4) or $\left(4^{\prime}\right)$ is considered here; that for vorticity $(3)$ or $\left(3^{\prime}\right)$ is similar. In methods I and V, the unknown temperature $\Theta_{i, j}^{n+1}$ can be written as an explicit linear combination of computed values at time $n$ :

$$
\Theta_{i, j}^{n+1}=a_{1} \Theta_{i+1, j}^{n}+a_{2} \Theta_{i-1, j}^{n}+a_{3} \Theta_{i, j}^{n}+a_{4} \Theta_{i, j+1}^{n}+a_{5} \Theta_{i, j-1}^{n}
$$

where the $a_{k}$ denote coefficients which vary in time, but which are constant over a time step. The essential feature of the method is to require that the norm of the matrix of coefficients $a_{k}$ at all times is bounded by unity. The row norm is used, and leads to

$$
\max _{i, j}\left\{\sum_{k=1}^{5}\left|a_{k}\right|\right\} \leqslant 1
$$

where $\max _{i, j}$ denotes the maximum value of the quantity in braces for all $i, j$ in the grid system. Stability in the sense of Lax and Richtmyer [9, 14 (see p. 44)] exists if inequality (17) is satisfied. For methods I and V, satisfying (17) is equivalent to requiring that all the coefficients $a_{k}$ are positive. This follows because for methods $\mathrm{I}$ and $\mathrm{V}$ the coefficient $a_{3}$ has the special form:

$$
a_{3}=1-\left\{a_{1}+a_{2}+a_{4}+a_{5}\right\}
$$

which can be readily verified. The conditions under which the coefficients $a_{k}$ are positive for methods $\mathrm{I}$ and $\mathrm{V}$ are discussed next.

For method I, the coefficients $a_{k}(k=1,2,4,5)$ are always positive, whereas $a_{3}$ can be made positive by restricting the size of the time step $\Delta \tau$. For $\operatorname{Pr} \leqslant 1$, the most severe restrictions on $\Delta \tau$ are:

$$
\begin{aligned}
& \Delta \tau \leqslant\left[\frac{\left|U_{i, j}\right|}{\Delta X}+\frac{\left|V_{i, j}\right|}{\Delta R}+\frac{2}{(\Delta X)^{2}}+\frac{2}{(\Delta R)^{2}}\right]^{-1}, \\
& \Delta \tau \leqslant\left[\frac{\left|U_{i, 0}\right|}{\Delta X}+\frac{2}{(\Delta X)^{2}}+\frac{4}{(\Delta R)^{2}}\right]^{-1}
\end{aligned}
$$

and

$$
\Delta \tau \leqslant\left[\frac{\left|U_{i, 0}\right|}{\Delta X}+\frac{2 P r}{(\Delta X)^{2}}+\frac{8 P r}{(\Delta R)^{2}}\right]^{-1}
$$

These restrictions respectively follow by requiring that $a_{3}$ be positive for the finite-difference representations of eqs $\left(4^{\prime}\right),\left(7^{\prime} b\right)$, and $\left(7^{\prime} a\right)$.

For method V (as for method I above), the coefficients $a_{k}(k=1,2,4,5)$ are always positive. In order to make $a_{3}$ positive, however, several different restrictions on $\Delta \tau$ arise because method $\mathrm{V}$ employs forms for the convection terms that depend upon the sign of the mean velocities, as noted in table 2. For the case when the mean velocities $\bar{U}_{i, j}, \bar{U}_{i-1, j}, \bar{V}_{i, j}$ and $\bar{V}_{i, j-1}$ are positive ${ }^{7}$ and $\operatorname{Pr} \leqslant 1$, the greatest restrictions on $\Delta \tau$ result from the difference forms of the temperature equations (4) and (7):

$$
\Delta \tau \leqslant\left[\frac{\bar{U}_{i, j}}{\Delta X}+\left(1+\frac{1}{2 j}\right) \frac{\bar{V}_{i, j}}{\Delta R}+\frac{2}{(\Delta X)^{2}}+\frac{2}{(\Delta R)^{2}}\right]^{-1}
$$

${ }^{7}$ The mean velocities are defined by

$$
\bar{U}_{m, n}=\frac{U_{m+1, n}+U_{m, n}}{2} \text { and } \bar{V}_{m, n}=\frac{V_{m, n+1}+V_{m, n}}{2}
$$


and

$$
\Delta \tau \leqslant\left[\frac{\bar{U}_{i, 0}}{\Delta X}+4 \frac{\bar{V}_{i, o}}{\Delta R}+\frac{2}{(\Delta X)^{2}}+\frac{4}{(\Delta R)^{2}}\right]^{-1}
$$

Additional forms similar to (20) arise when the mean velocities are negative, or a combination of positive and negative.

Some observations on the application of the stability requirements can be noted. It is impractical to alter $\Delta X$ and $\Delta R$ according to eqs (15a) during a computation to achieve stability for methods II-IV. Accordingly, a cut and try procedure is employed. The time step, however, is adjusted during a computation with all methods, using equations (15b), (15c), (19), or (20), as appropriate. The field of mesh points is scanned in order to determine the largest allowable $\Delta \tau$ which will satisfy the stability requirements. Time steps smaller than this value can be used, of course, to provide a safety factor. In practice, 80 percent of the allowable $\Delta \tau$ was used with methods I-IV and 95 percent with method V.

\section{Comparative Physical Results}

Natural convection flows calculated with the five numerical methods are presented and compared in sections 4.1 through 4.3 for a Grashof number of $G r=1 \times 10^{5}$. The calculations employed a uniform grid spacing of $\Delta X=\Delta R=0.05$, with a total of 121 mesh points. The calculation of physically meaningful flows at higher values of $G r$, and some questions of convergence, are considered in section 4.4 .

Flow patterns and temperature fields are illustrated in figures 2 through 6 with computerdrawn graphs displaying sets of streamlines and isotherms. The location of these streamlines and isotherms was determined by linear interpolation of the computed values at the mesh points. In each of the graphs, the centerline of the cylindrical enclosure is shown on the left. The abscissa is the radial coordinate $R$, and the ordinate is the axial coordinate $X$. The heat source on the floor is denoted with a thick line between $R=0$ and $R=0.1$.

\subsection{Transient Streamline and Temperature Fields}

All the numerical calculations were carried from the initial quiescent condition forward in time until steady state was achieved. The transient and steady-state flows calculated during this process are qualitatively similar for all five numerical methods. The purpose of this section is to discuss the physical nature of the flows, consequently, only the transient results for one method need be considered. Method V is considered representative, and the transient flow and temperature fields calculated with this method are illustrated in figures 2 and 3, respectively. These figures are each composed of four graphs, arranged in order of increasing time, $\tau$.
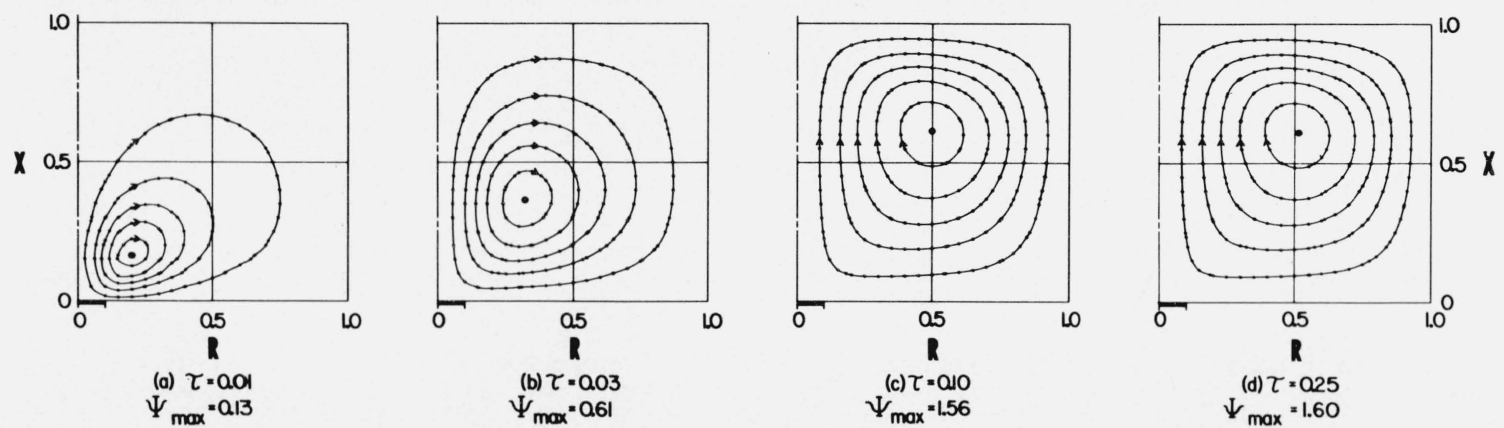

FigURE 2. Transient streamline fields calculated with method $\mathrm{V}$ at various times $(\tau), \mathrm{Gr}=1 \times 10^{5}$.

Streamline evolution is qualitatively similar for all five numerical methods. The walls and centerline correspond to $\Psi=0$; the dot has value $\Psi_{\text {max }}$; the remaining streamlines correspond successively to $\Psi$ values of $0.1,0.3,0.5,0.7$, and 0.9 , of $\Psi_{\max }$. 


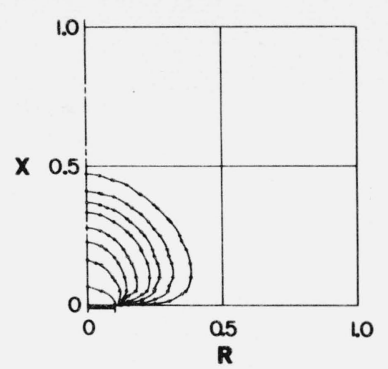

(a) $\tau=0.01$

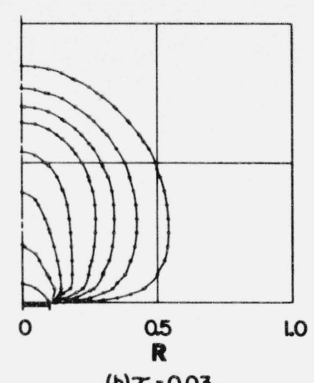

(b) $\tau=0.03$

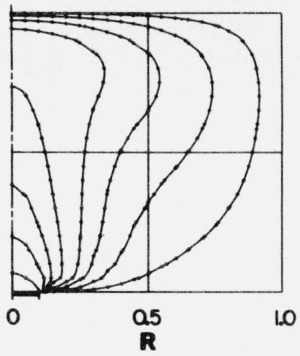

(c) $\tau=0.10$

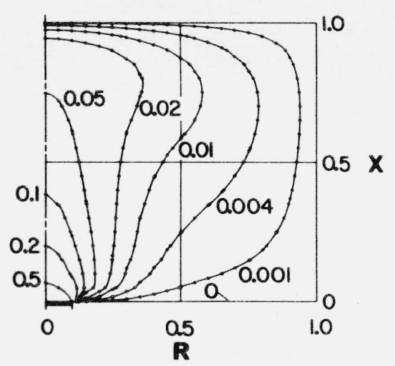

(d) $\tau=0.25$

FIGURE 3. Transient temperature fields calculated with method $\mathrm{V}$ at various times $(\tau), \mathrm{Gr}=1 \times 10^{5}$.

Temperature evolution is qualitatively similar for all five numerical methods. Temperature $(\theta)$ is the curve parameter; the heat source (thick line on floor) is at $\theta=1$.

Immediately after the start of heating, a ring vortex of warm fluid forms near the origin (figs. $2 \mathrm{a}$ and $3 \mathrm{a}$ ). This vortex rises and moves radially outward from the centerline (figs. $2 \mathrm{~b}$ and $2 \mathrm{c}$ ). A gradual development into the steady state flow and temperature fields then follows. The steady state fields reveal a ring vortex centered just above $X=0.5, R=0.5$ (fig. $2 \mathrm{~d}$ ) which is driven by the heated fluid which rises along the centerline from the floor to the ceiling (fig. $3 \mathrm{~d}$ ).

A sensitive indication of the approach to steady state was provided by the overall energy balance on the enclosure. When the heat transfer rates into and out of the enclosure were within 1 percent of an asymptotic value, steady conditions prevailed. Steady state was achieved by $\tau=0.25$ with all methods.

\subsection{Comparison of Steady-State Streamline and Temperature Fields}

The qualitative similarity of flows calculated with the five methods was mentioned in the last section. The purpose of the present section is to illustrate this similarity in a quantitative way, by comparing steady state flows obtained with methods I-V. Such a comparison of the steady-state streamline and temperature fields is presented in figures 4 and 5 , respectively. The sets of streamlines and isotherms shown have the same numerical values as in figures 2 and 3 . Figures 4 and 5 each contain four individual graphs, which respectively pertain to numerical methods I, II, III, and V. Computed results from methods III and IV agreed to at least three significant figures, and graphs of the steady state streamline and temperature fields for the two methods are identical. Consequently, only the results of method III are shown. Figures $4 \mathrm{~d}$ and $5 \mathrm{~d}$ duplicate figures $2 \mathrm{~d}$ and $3 \mathrm{~d}$ respectively.
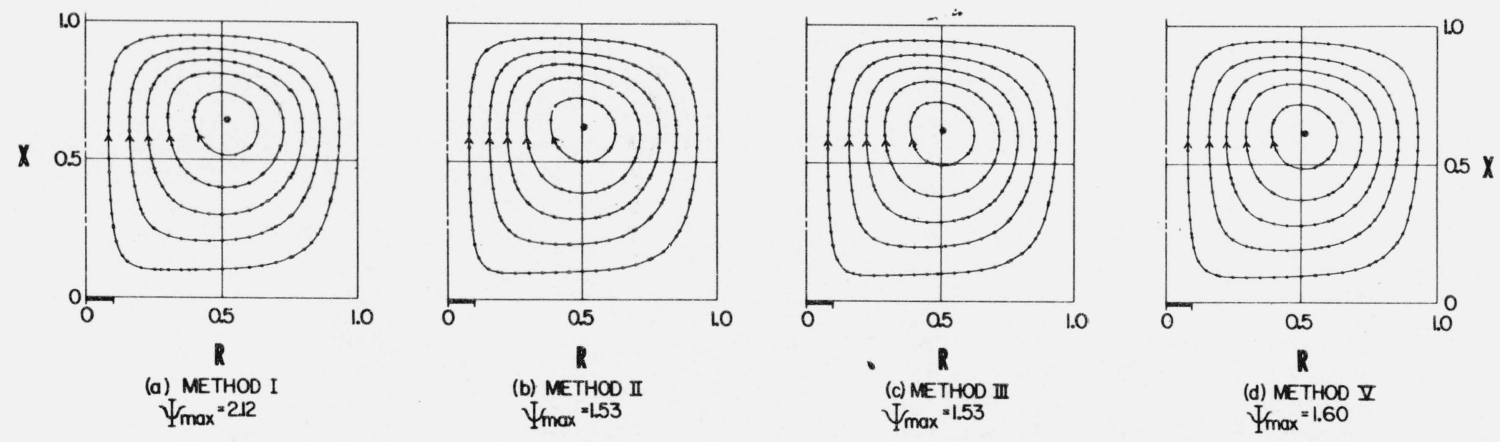

FigurE 4. Steady-state streamline fields for the various numerical methods, $\mathrm{Gr}=1 \times 10^{5}$.

Results for methods III and IV are identical. The walls and centerline correspond to $\Psi=0$; the dot has value $\Psi_{\max }$; the remaining streamlines correspond successively to $\Psi$ values of $0.1,0.3,0.5,0.7$, and 0.9 , of $\Psi_{\max }$. 


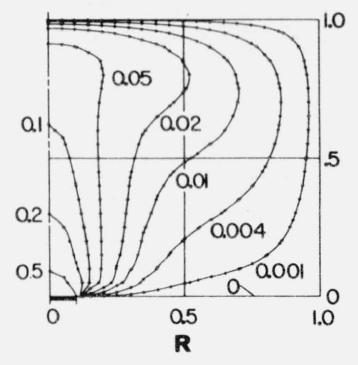

(a) METHOD I

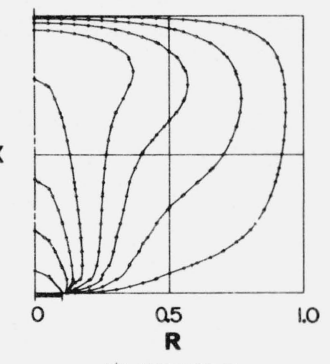

(b) METHOD II

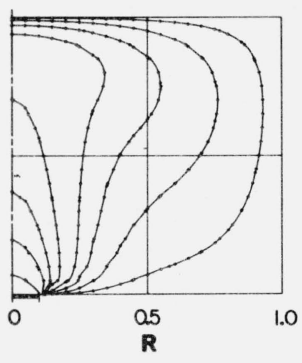

(c) METHOD III

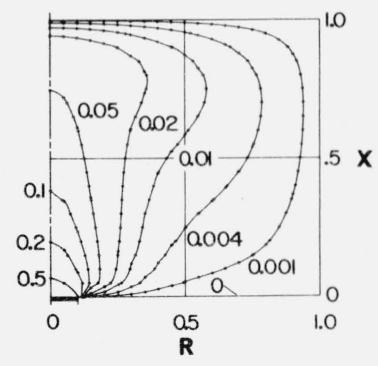

(d) METHOD Z

FigURE 5. Steady state temperature fields for the various numerical methods, $\mathrm{Gr}=1 \times 10^{5}$. Results for methods III and IV are identical. Temperature $(\theta)$ is the curve parameter; the heat source (thick line on floor) is at $\theta=1$.

A comparison of figures $4 a^{-}-4 d$ or $5 a^{-}-5 d$ reveals a striking similarity in the streamline and temperature fields computed with the various numerical methods. Method I shows the greatest departure from the group with the largest value of $\Psi_{\max }$ (caption, fig. 4a) and in the shape of the isotherms above the heat source (fig. 5a). The physical reason for this is that method I transfers more heat by convection from the heat source than the other methods (and thus has the greatest rate of fluid circulation, $\left.\Psi_{\max }\right)$. This is discussed further at the end of section 4.3.

Figures 4 and 5 illustrate flows in which both convective and diffusive transport exists. The contribution of convection to the temperature fields in figure 5 can be appraised by referring to figure 6. This figure illustrates the steady state temperature field for static conduction (no fluid motion) and was obtained with method V by setting $G r=U=V=\Omega=\Psi=0$ in the basic differential equations (1)-(4). The conduction fields calculated in this way for numerical methods $\mathrm{I}-\mathrm{V}$ are identical (to at least four significant figures) because all methods approximate the heat diffusion (or conduction) terms to the same spatial accuracy.

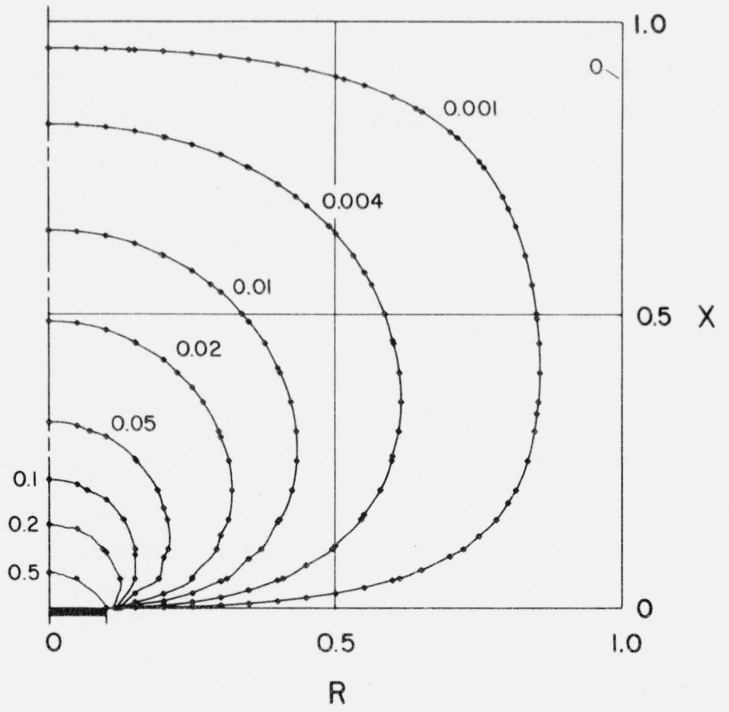

FiguRE 6. Static conduction temperature field at steady state, all methods.

Temperature $(\theta)$ is the curve parameter; the heat source (thick line on floor) is at $\theta=1$.

Table 3 lists the computer time (in seconds) required to calculate the flows illustrated in figures 4 and 5. The particular computer used had execution times of 1.75 and $2.625 \mu$ s for nine digit floating point addition and multiplication, respectively. The only significant observation that can be made about this table is that method III required the least computer time, and method I the most. 
Differences between methods could be reduced by altering the safety factor used for the time step $\Delta \tau$ or by more efficient programming.

TABLE 3. Computer time required for test case with $\mathrm{Gr}=1 \times 10^{5}$ and a $21 \times 21$ mesh

\begin{tabular}{|c|c|}
\hline Method & Computer time \\
\hline & sec \\
\hline I... & 335 \\
\hline II...... & 270 \\
\hline III..... & 230 \\
\hline IV ...... & 309 \\
\hline $\mathrm{V} \ldots \ldots$. & 285 \\
\hline
\end{tabular}

\subsection{Heat Transfer Rates}

The heat transfer rates calculated by the five numerical methods are conveniently discussed in terms of the rate of heat addition $\left(\dot{Q}_{\text {in }}\right)$ or heat removal $\left(\dot{Q}_{\text {out }}\right)$ from the enclosure. These are total quantities, obtained by integration over the areas of the heat source and cold walls, respectively. The integration is performed by computing the heat transfer by convection and conduction from wall mesh points to adjacent mesh points, as discussed in the paragraph at the end of section $3.2 \mathrm{a}$. A dimensionless heating rate can be defined as

$$
\Phi=\frac{\dot{Q}}{\lambda a \Delta T}
$$

where $\lambda$ is the thermal conductivity of air, $a$ is the height of the enclosure, and $\Delta T$ is the imposed temperature difference driving the flow, $\Delta T=T_{h}-T_{0}$. Equation (21) defines a dimensionless heat addition $\left(\Phi_{\text {in }}\right)$ or heat removal $\left(\Phi_{\text {out }}\right)$ as the rate of heat transfer is $Q_{\text {in }}$ or $Q_{\text {out }}$, respectively.

The heat transfer rates discussed in this section pertain to a ramp temperature change at the edge of the heat source. This ramp is the grid approximation of the boundary conditions (6) (a step change) and appears as a linear variation of temperature along the floor from $\theta=1$ at $R=R_{b}-\Delta R / 2$ to $\Theta=0$ at $R=R_{b}+\Delta R / 2$. Both numerically and experimentally, a step change in temperature is difficult to achieve. The rate of heat transfer, while being finite for a ramp, would be infinite for a step. Twenty-one radial mesh points were used throughout this study and an extension of this study [18]. The corresponding ramp (see fig. 4 of [18]) is a close approximation of the floor temperature profile in a physical experiment [17] with which the numerical flows could be compared.

Curves illustrating the heat transfer rates for numerical methods I and II as a function of time are presented in figure 7; similar results for methods III-V are presented in figure 8 . Figures 7 and 8 illustrate nonconservation and conservation methods, respectively. The ordinate is the dimensionless heat transfer rate into $\left(\Phi_{\text {in }}\right)$ or out of $\left(\Phi_{\text {out }}\right)$ the enclosure. The abscissa is the dimensionless time $\tau$.

The calculated heat transfer rates due to static conduction $(G r=0)$ are virtually identical for all five numerical methods. These conduction curves are shown in both figures 7 and 8 as a basis for comparison. Note that the heat removed (dashed line) approaches the heat added (solid line) with increasing time. Thus, the finite-difference approximation of the diffusion terms for all methods conserves energy within the grid system.

Methods I and II, figure 7, employ difference approximations of the convection terms which do not conserve energy. For method I, the rate of heat removal exceeds the rate of heat addition at steady state, indicating a net production of heat within the enclosure. For method II, the heat addition exceeds the heat removal, indicating a net absorption of heat within the enclosure. Clearly, 


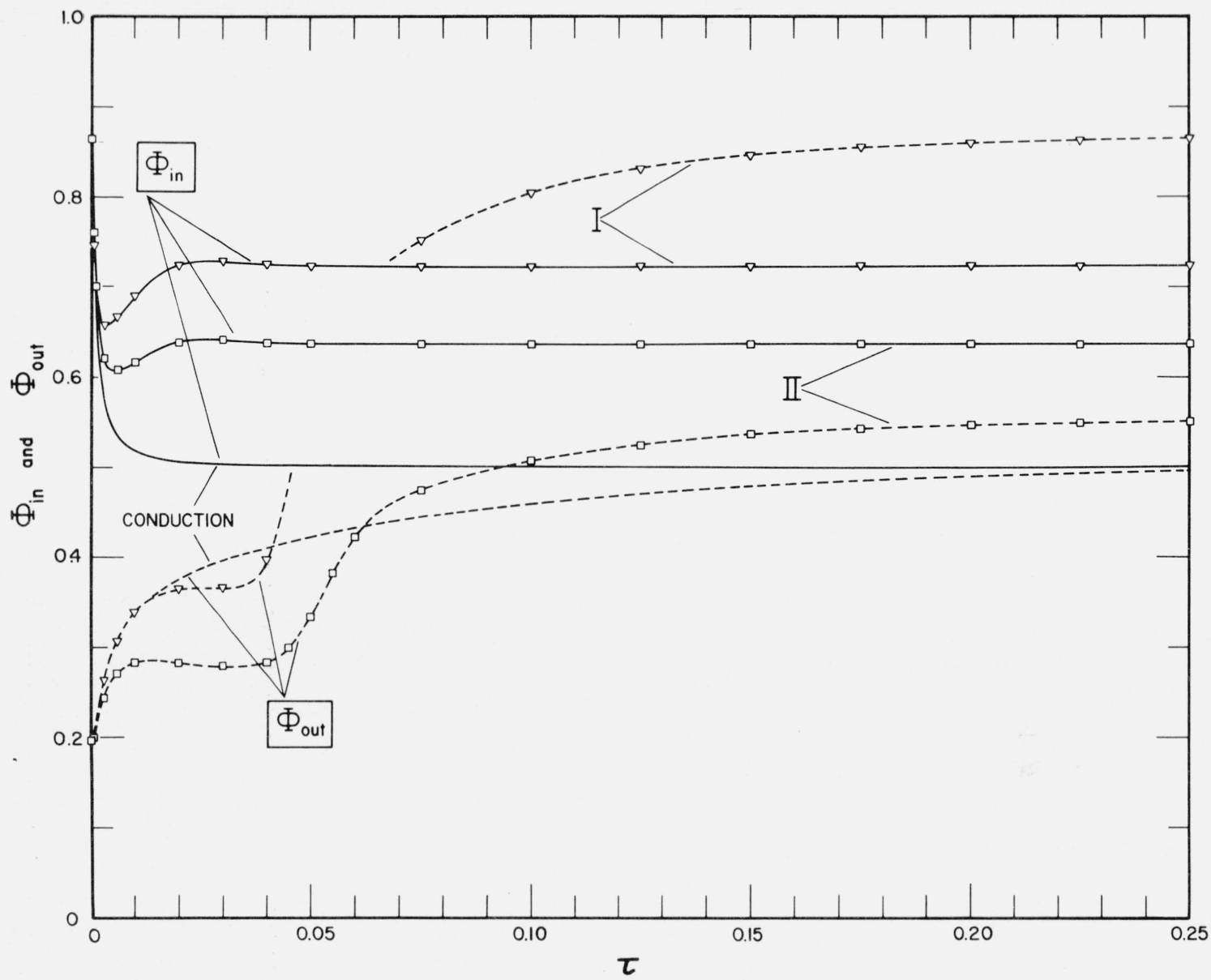

FIGURE 7. Dimensionless heat flow into $\left(\Phi_{\text {in }}\right)$ and out of $\left(\Phi_{\text {out }}\right)$ the enclosure as a function of time $(\tau)$ for methods I and II $\left(\mathrm{Gr}=1 \times 10^{5}\right)$ and static conduction (all methods).

methods I and II violate the laws of thermodynamics. These differences in the rates of heat addition and heat removal are due to residues which remain when the difference equations for temperature are summed over all interior grid points, and the convection terms do not cancel in pairs. Methods I and II thus do not retain the energy conservation expressed by the basic differential equations. ${ }^{8}$

Methods III through V, figure 8, employ difference approximations of the convection terms which conserve energy. Clearly, for each method the rate of heat addition equals the rate of heat removal at steady state. Methods III and IV employ derivative approximations with similar spatial accuracy. As a consequence, the heat transfer curves for these methods are virtually identical.

Two general observations about figures 7 and 8 can be made. First, the shape of the heat addition curves is qualitatively similar for all methods, as is the shape of the heat removal curves. All curves for heat addition (solid lines) follow the conduction curve initially, then break away and achieve a steady value by $\tau=0.05$. The heat removal curves (dashed lines) also follow the conduction curve initially. The heat removal rate then falls below the conduction value as it becomes more difficult for heat to be conducted radially from the heat source to the nearby cold floor counter to the incoming flow. At about $\tau=0.04$, the rate of heat removal begins to increase sharply as the

${ }^{8}$ The authors of methods I [2] and II [20] did not calculate the heat transport from node to node within the mesh system. Instead, four point difference formulas were employed to approximate the temperature gradient at the walls, and thus the rates of heat transfer. For unsymmetric heating, this approach does not lead to equality between the steady state rates of heat removal and heat addition. 


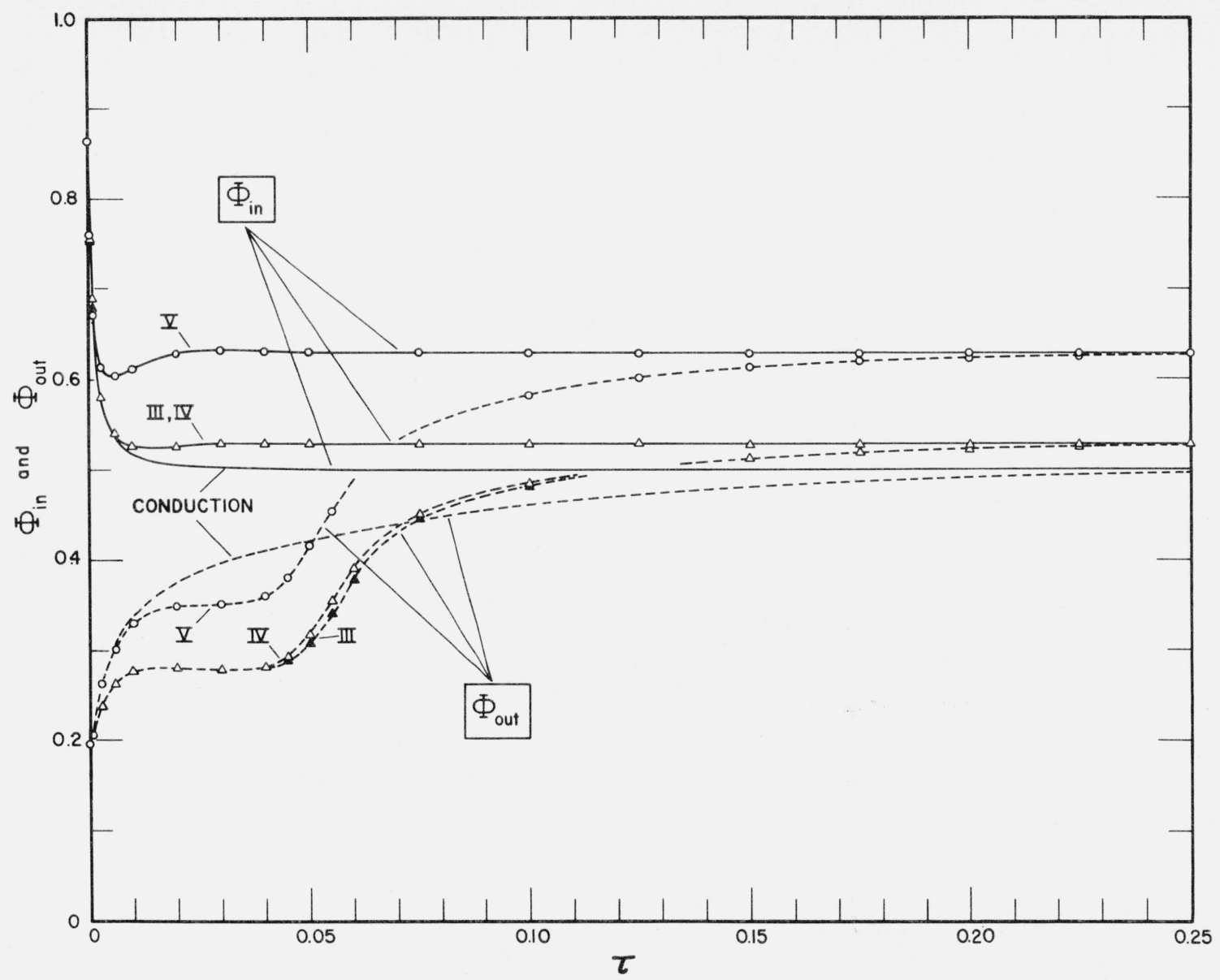

FigURE 8. Dimensionless heat flow into $\left(\Phi_{\text {in }}\right)$ and out of $\left(\Phi_{\text {out }}\right)$ the enclosure as a function of time $(\tau)$ for methods III, IV and $V\left(\mathrm{Gr}=1 \times 10^{5}\right)$ and static conduction (all methods).

heated fluid reaches the ceiling above the hot spot. As the fluid spreads radially out ward along the ceiling, the rate of heat removal continues to rise. The lower curve then gradually approaches the upper (in fig. 8).

The second general observation that can be made about figures 7 and 8 concerns the magnitude of the steady-state heat additions. Clearly, the difference between the values for the five methods at $G r=1 \times 10^{5}$ and the value for conduction is a measure of the convective heat transfer from the heat source. This convective contribution decreases for the five methods in the sequence: method $\mathrm{I}>\mathrm{II}>\mathrm{V}>\mathrm{III}=\mathrm{IV}$. The relative magnitudes of these contributions can be estimated by applying the difference approximation of the convection terms $U \partial \theta / \partial X$ or $\partial(U \Theta) / \partial X$ (table 2) to the grid point $(i=1, j=0)$. A portion of this difference approximation is associated with the convective transport of energy from grid point $(i=0, j=0)$ to $(i=1, j=0)$. A careful study reveals that the convective heat transport for the five methods is proportional to the following quantities: method I $\propto U_{1,0} ;$ II $\propto U_{1,0}\left(1+\Theta_{1,0}\right) / 2 ; \mathrm{V} \propto U_{1,0} / 2 ;$ and III and IV $\propto U_{1,0} \Theta_{1,0} / 2$. (The boundary conditions $U_{0,0}=0$ and $\Theta_{0,0}=1$ were employed.) The temperature $\Theta_{1,0}$ lies between 0 and 1 ; thus, the aforementioned sequence of methods is in order of decreasing convection. This agrees with the results in figures 7 and 8.

\subsection{Extension to Higher Gr, Convergence}

The calculation of flows at high Grashof numbers is considered in this section. Results at $G r=1 \times 10^{5}$ in sections 4.1 and 4.2 revealed a qualitative similarity in the calculated flows for the 
five numerical methods. Extension to higher $G r$, however, means that the methods must continue to produce physically meaningful solutions, and this is closely associated with the practical stability, truncation errors, and conservation properties discussed in sections 3 and 4.3. For conservation reasons alone, we discard methods I and II from further consideration, and consider only the conservation methods III-V.

Of the latter methods, III and IV impose a spatial mesh size restriction to achieve stability while $\mathrm{V}$ does not. Violation of this restriction leads to oscillations in the fields of $\Theta, \Psi, U$, and $V$. The effect of violating the spatial mesh size restriction is illustrated in figure 9 for the steady-state centerline temperature at $G r=1 \times 10^{6}$. The ordinate is the axial coordinate $X$ and the abscissa is the temperature $\Theta$. The curves on the right pertain to method III with the number of vertical mesh points, $M$, equal to 21, 41, and 61. (Results for methods III and IV were identical.) For comparison, results from method $\mathrm{V}$ are shown on the left with $M=21,51$, and 101 . In all cases, the number of radial mesh points, $N$, is 21 .

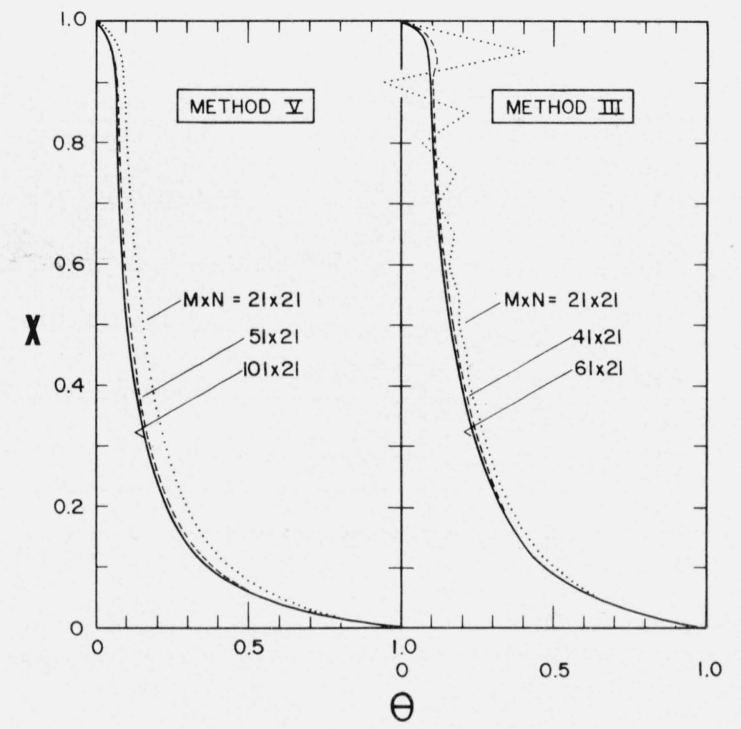

FIGURE 9. Steady state distribution of temperature $(\Theta)$ with height $(\mathrm{X})$ along the centerline; methods $I I I$ and $V, \mathrm{Gr}=1 \times 10^{6}$. Curve parameter is the number of vertical mesh points $(M)$.

From figure 9 , it is immediately apparent that method III leads to an oscillating temperature near the ceiling $(X=1)$ when $M=21$. Repeating the calculation with $M=41$ reduces the oscillation, but a local maximum persists at $X=0.95$. Further refinement to $M=61$ eliminates this hot spot. With 21 and 41 vertical mesh points, occasional negative temperatures were calculated during the transient. The steady state results for $M=21$ reveal a region of negative temperature at $X=0.8$. With $M=11$, register overflows occurred early in the transient, and a stable integration was not possible.

With method V, no such oscillations or negative temperatures were calculated. Increasing the number of vertical mesh points from 21 to 101 reveals that the temperature distribution appears to asymptotically approach a limiting curve. Note that the asymptotic temperature distributions for methods III and V are not identical; this is attributed to differences in the truncation errors.

Oscillations such as those in figure 9 are physically unrealistic, and appear only when the mesh size restrictions (inequalities (15a)) are not satisfied. Such oscillations have not been observed with method V. The inequalities (15a) are satisfied for the test case at $G r=1 \times 10^{5}$ with a $21 \times 21$ mesh. As the Grashof number is increased, it has been found for the present problem [18] that $U_{\max } \propto G r^{1 / 2}$. Clearly, through (15a), this quickly leads to a very fine grid and prohibitively large computer storage requirements. 
The freedom of method $\mathrm{V}$ from a spatial mesh size restriction (and from oscillations) suggests its application to high $G r$ flows. Due to truncation errors which increase with $G r$, such results must be interpreted carefully. The solutions should be tested for convergence or compared with physical experiment; two such tests for method V are discussed in the subsequent paragraphs.

A type of convergence check is illustrated in figure 10. The ordinate is the steady state heat flow into the enclosure, $\Phi_{\text {in }}$, and the abscissa is the number of vertical mesh points, $M$. The curve parameter is the Grashof number. Solid lines pertain to method V, a single dashed line is shown for method III. For reasons noted at the beginning of section 4.3, the number of radial mesh points was held fixed at $N=21$ (except for the one solid data point for which $N=31$ ). The dotted line is an analytical result [8] for conduction of heat from a heated disk into a semi-infinite medium. The disk has a temperature profile quite close to the ramp profile of the grid approximation. The numerical results for static conduction are reasonably close to the analytical conduction value. For convective flows at $G r=1 \times 10^{5}, 1 \times 10^{6}$, and $4 \times 10^{7}$, the numerical results tend toward an asymptote with increasing $M$.

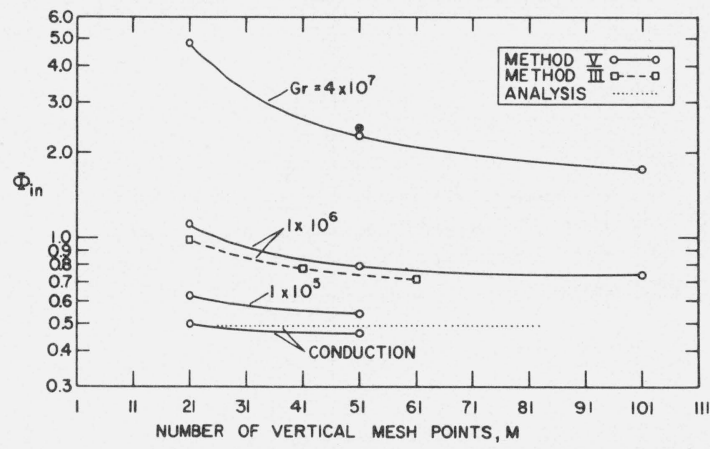

FIGURE 10. Steady state dimensionless heat flow $\left(\Phi_{\text {in }}\right)$ versus number of vertical mesh points $(\mathbf{M})$. Number of radial mesh points $(N)$ held fixed at $N=21$ (except for one solid data point for which $N=31$ ).

Additional support for the use of method V is provided by comparison of a numerically calculated streamline field with physical experiment. An example of such a comparison is presented in figure 11 at $G r=4 \times 10^{6}$. The numerical results are from an extension of this study [18], which employed a $51 \times 21$ grid $(M \times N)$ and covered a range of $G r$ from $4 \times 10^{4}$ to $4 \times 10^{10}$. The experimental results are from reference [17] which covered a range of $\mathrm{Gr}$ from $8 \times 10^{5}$ to $1 \times 10^{10}$. For $\mathrm{Gr}$ up to about $1 \times 10^{9}$, the comparison between numerical and physical experiment is very good. Above this value, the physical experiment indicates turbulence. The numerical experiment was laminar (presumably due to false viscosity and the assumed two-dimensional motion) up to $G r=4 \times 10^{10}$. At this value, the numerical flow developed a periodic vortex shedding, suggestive of the onset of laminar instability. This vortex shedding was adequately resolved with the grid spacing used.

A significant consideration in the application of any finite-difference procedure to a flow problem is the amount of computer time required to reach a steady state. Method V was applied in the numerical study of reference [18], and the computing time is shown in figure 12. Clearly, as $G r$ increases, the amount of computing time also increases (as $G r^{1 / 4}$ at large $G r$ ). Approximately one-half second was required for each time step, but for large $G r$ the size of the time step decreases rapidly and many more steps are required. 

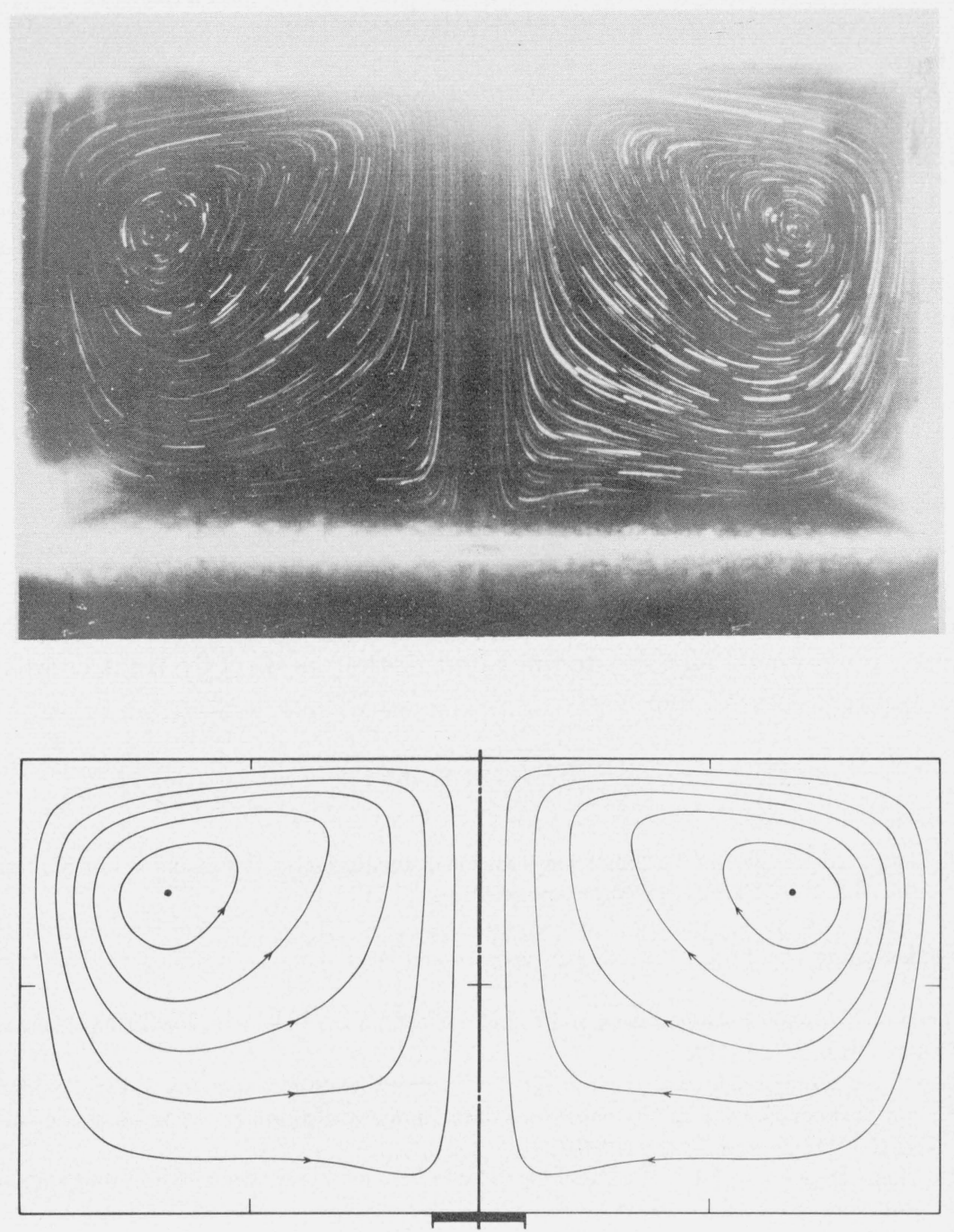

FIGURE 11. Comparison of numerical results of method $V$ (from reference [18]) with physical experiment (from reference [17]) at $G r=4 \times 10^{6}$.

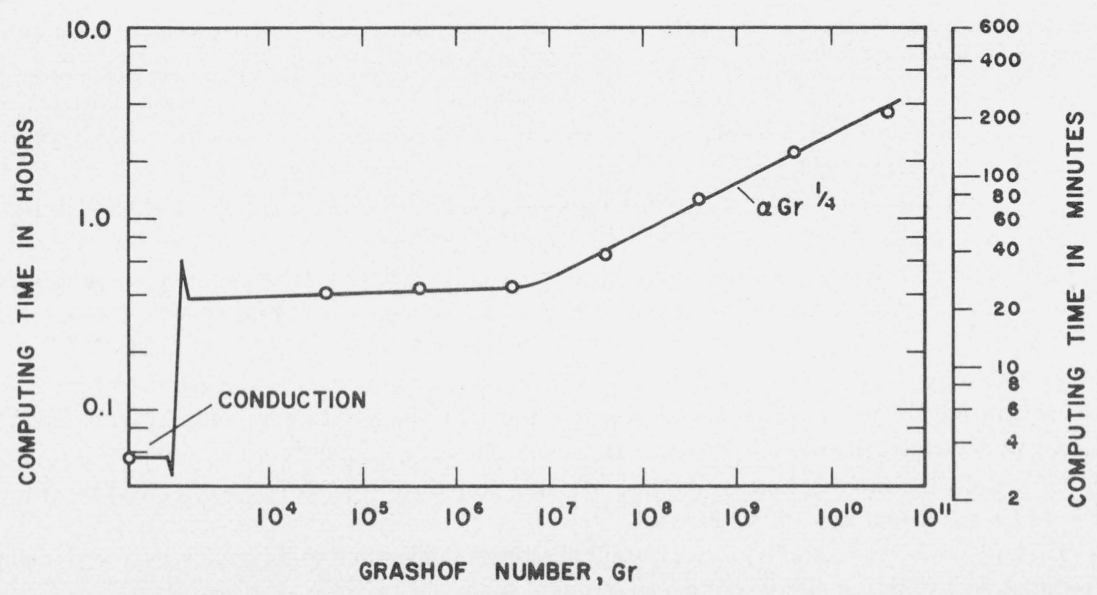

FigurE 12. Computing time required for method $V$ at several Grashof numbers (Gr), numerical study of reference [18]. Calculations carried from quiescent initial conditions to steady state with a $M \times N=51 \times 21$ grid. 


\section{Concluding Remarks}

Five finite difference procedures were compared for calculating transient natural convection flows in an enclosure. Although the problem was formulated in axisymmetric cylindrical coordinates, the conclusions should be applicable to any two-dimensional coordinate system.

To achieve stability of the time and space integrations, restrictions are imposed on the size of the time step for all methods (i.e., I-V) and on the size of the mesh spacing for methods II-IV.

Energy and vorticity are not conserved within the grid system with methods I and II, but are conserved with methods III-V. For this reason, the latter methods are to be preferred.

Of the conservation methods, method III appears to require less computer time than methods IV and V (table 3). Method III is therefore preferable when its associated mesh size restriction can be satisfied. If this restriction cannot be satisfied (which is usually the case) oscillations in the flow develop. A method free of a mesh size restriction (and oscillations) is then recommended, method V. The flows calculated with method $\mathrm{V}$ must be interpreted carefully, however, due to the truncation errors.

The author is indebted to L. Orloff, Factory Mutual Research Associate at the NBS, for developing a computer program to plot streamlines and isotherms directly from computed numerical fields of stream function and temperature.

\section{References}

[1] Arakawa, A., Computational design for long-term numerical integration of the equations of fluid motion: two-dimensional incompressible flow. Part 1, J. Computational Phys. 1, 119 (1966).

[2] Barakat, H. Z., and Clark, J. A., Analytical and experimental study of the transient laminar natural convection flows in partially filled liquid containers, Proc. Third International Heat Transfer Conf. (Chicago, Illinois) Vol. II, 152 (1966).

[3] Bryan, K., A scheme for numerical integration of the equations of motion on an irregular grid free of nonlinear instability, Monthly Weather Rev. 94, 39 (1966).

[4] Chandrasekhar, S., Hydrodynamic and Hydromagnetic Stability (Oxford University Press, London, England, 1961).

[5] DuFort, E. C., and Frankel, S. P., Stability conditions in the numerical treatment of parabolic equations, Math. Tables and Other Aids to Computation 7, 135 (1953).

[6] Fromm, J., The Time Dependent Flow of an Incompressible Viscous Fluid, Methods in Computational Physics 3, 345 (Academic Press, New York, N.Y., 1964).

[7] Hellums, J. D., and Churchill, S. W., Computation of natural convection by finite difference methods, International Developments in Heat Transfer-Proc. of the 1961-62 International Heat Transfer Conf. (Boulder, Colorado and London, England), 985 (1963).

[8] Karush, W., and Young, G., Temperature rise in a heat-producing solid behind a surface defect, J. Appl. Phys. 23, 1191 (1952).

[9] Lax, P. D., and Richtmyer, R. D., Survey of the stability of linear finite difference equations, Communications on Pure and Appl. Math. 9, 267 (1956).

[10] Milne-Thomson, L. M., Theoretical Hydrodynamics (Macmillan, New York, N.Y., fourth edition, 1960).

[11] O'Brien, G. G., Hyman, M. A., and Kaplan, S., A study of the numerical solution of partial differential equations, J. Math. and Phys. 29, 223 (1950).

[12] Peaceman, D. W., and Rachford, H. H., Jr., The numerical solution of parabolic and elliptic differential equations, J. Soc. Indust. Appl. Math. 3, 28 (1955).

[13] Richardson, L. F., The approximate arithmetical solution by finite differences of physical problems involving differential equations, with an application to the stresses in a masonry dam, Phil. Trans. R. S. London 210A, 307 (1910).

[14] Richtmyer, R. D., Difference Methods for Initial Value Problems (Interscience, New York, N.Y., 1957).

[15] Runchal, A. K., Spalding, D. B., and Wolfshtein, M., The numerical solution of the elliptic equations for transport of vorticity, heat and matter in two-dimensional flows, Imperial College (London), Dept. Mech. Eng., SF/TN/2 (1967).

[16] Todd, J., Survey of Numerical Analysis (McGraw-Hill, New York, N.Y., 1962).

[17] Torrance, K. E., Orloff, L., and Rockett, J. A., Experiments on natural convection in enclosures with localized heating from below, to be published in J. Fluid Mech.

[18] Torrance, K. E., and Rockett, J. A., Numerical study of natural convection in an enclosure with localized heating from below-creeping flow to the onset of laminar instability, to be published in J. Fluid Mech.

[19] Wilkes, J. O., The finite difference computation of natural convection in an enclosed rectangular cavity (Ph. D. thesis, Univ. Michigan, Ann Arbor, 1963). 
[20] Wilkes, J. O., and Churchill, S. W., The finite-difference computation of natural convection in a rectangular enclosure, Am. Inst. Chem. Eng. J. 12, 161 (1966).

[21] Young, D., Iterative methods for solving partial difference equations of elliptic type, Trans. Am. Math. Soc. 76, 92 (1954).

(Paper 72B4-279) 